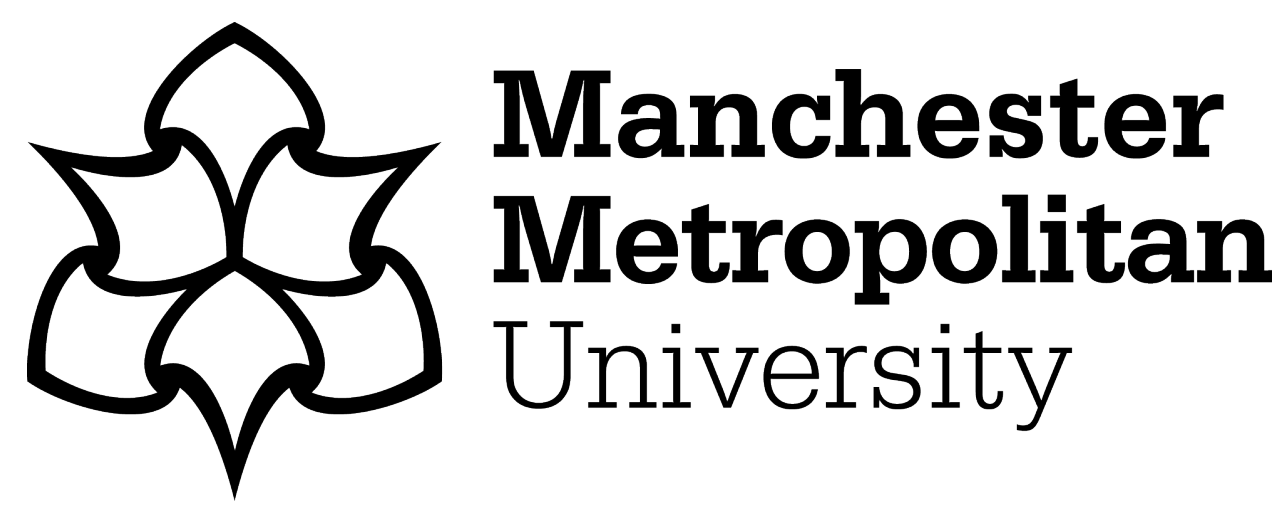

Gheth, W, Alfitouri, A, Rabie, Khaled ORCID logoORCID: https://orcid.org/0000-0002-9784-3703, Adebisi, B ORCID logoORCID: https://orcid.org/0000-0001-9071-9120 and Hamdi, Khairi Ashour (2019) Performance Analysis of Cooperative Diversity in Multi-user Environments. In: 8th International Conference on Modeling Simulation and Applied Optimization (ICMSAO), 15 April 2019 - 17 April 2019, Manama, Bahrain.

Downloaded from: https://e-space.mmu.ac.uk/622872/

Version: Accepted Version

Publisher: IEEE

DOI: https://doi.org/10.1109/ICMSAO.2019.8880443

Please cite the published version 


\title{
Performance Analysis of Cooperative Diversity in Multi-user Environments
}

\author{
Waled Gheth ${ }^{1}$, A. Alfitouri ${ }^{2}$, Khaled M. Rabie ${ }^{1}$, Bamidele Adebisi ${ }^{1}$ and Khairi Ashour Hamdi ${ }^{3}$ \\ ${ }^{1}$ School of Engineering, Manchester Metropolitan University, Manchester, UK \\ ${ }^{2}$ School of Electrical and Electronic Engineering, Engineering Academy Tajoura, Tripoli, Libya \\ ${ }^{3}$ School of Electrical and Electronic Engineering,The University of Manchester, Manchester, UK \\ Emails:\{w.gheth, k.rabie, b.adebisi\}@mmu.ac.uk; a.alfitouri@hotmail.com; k.hamdi@manchester.ac.uk
}

\begin{abstract}
The article studies the performance of cooperative multi-relay networks with random numbers of accessing users. A cooperative diversity is achieved at the destination nodes by receiving multiple independent copies of the same signal from $M$ relays when all relays participate in the second phase of data transmission. The overall spectral efficiency (SE) of the considered system is investigated and accurate analytical expressions for it are developed. Furthermore, the article discusses how system performance is affected by its parameters. Monte Carlo simulations are used to validate the analytical results. The results revealed that increasing relays number on the network can improve the system performance. The results also indicated that there was improvement in the performance when the number of users increased. However, the performance dropped when this number became close the relays number.
\end{abstract}

Index Terms-Cooperative communication, multi-relays system, spectral efficiency, zero forcing.

\section{INTRODUCTION}

This kind of cooperative technology has attracted the researchers" attention due to the dramatic performance gains that can be achieved in the case of the unavailability of the other types of diversity and when users share antennas and other resources to exchange channel information and transmitted symbols [1]. Transmission in wireless communication is mostly affected by shadowing, fading and distance between the source and the end-users, particularly when the communication is taking place between multiple-source nodes and multiple-destinations. In such cases, relying protocols can play a crucial role in aiding wireless communication by improving the performance of the system [2]. Different relaying protocols can be implemented, such as amplify-and-forward (AF) and decode-and-forward (DF), in order to improve the throughput and extend the coverage area without change the source power, particularly for the users at edge of the cell [3], [4]. There are some technical issues associated with the implementation of relays in wireless communication systems, which can degrade system performance. The most crucial one is signals interference at the relay. However, zero forcing (ZF) is one of the interference-cancellation techniques that can be applied at the source, relay and/or destination nodes, or any combinations of these to eliminate interference. This kind of scheme is a multi-node $\mathrm{ZF}$ protocol, where the distributed single antenna nodes can exchange channel information and can be used to apply ZF processing [5]-[7].

Cooperative distributed multi-users relaying an ad-hoc wireless network are discussed in [8], where the gain factors of the relays is derived to minimize the mean squared error (MSE) at the destination. Multi-way relaying in Rician fading channels is proposed in [9], where the authors reduced the transmission time by applying random and semi-orthogonal relay selection schemes. The study in [10] discussed the cooperative diversity performance over Nakagami-m fading channels utilizing equal gain combinations. The authors in [11] discussed the selection schemes of wireless network relay. They introduced different sub-optimal schemes with linear complexity for the optimal relay selection. Furthermore, the study investigated the achievable diversity orders of the network relay selection schemes.

In this paper, the performance of an AF multi-relay system is analyzed, where the communication between the random users $K$ and the destinations accrues via a number of relays $M$. All nodes are provided with a single antenna. We also discuss the effect of the users accessing number on the proposed cooperative multi-relay networks performance, where the relays communicate with several users in the same time-frequency resource to achieve a higher data rate. At the destination node we achieve cooperative diversity by receiving multiple independent copies of the same signal from $M$ relays, where all relays cooperate in the second stage of the communication process. Instead of adopting the best relay selection to forward data, we sum all the signals that come from all active relays.

Considering the fading and thermal noise effect at relays and end-user nodes, novel and accurate expressions are derived for multiple access interference at these nodes. So far, however, there has been little discussion about considering the communication between random users in wireless domain. The assumption of random users shows how the system performance is affected by the user activity and makes the derived expression more practicable than the existing ones. Further more, the ZF technique is applied at the relay nodes to tackle the interference. The derivation of the novel expressions can be utilized in the throughput of the multi-relays technique estimation in the considered channels. Such derivation, gives 


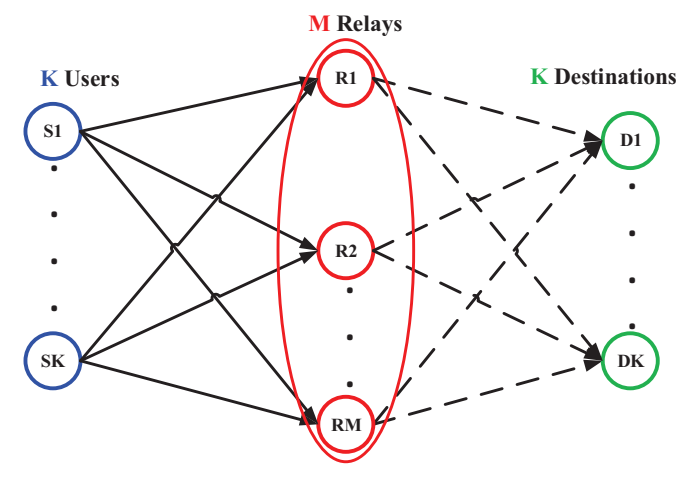

Fig. 1: System Model

us the opportunities to explore the effect of various system parameters on its performance. The analytical results of the new expressions are validated by computer simulations.

The rest of this article is as follows. The system model under consideration is presented in the following section. Section III presents the analysis of the over all capacity. The discussions of the numerical results are in Section IV. Finally, we present our main conclusions in Section V.

\section{SySTEM MOdEL}

The proposed system model is presented in Fig. 1. $K_{a}$ represents the random active users number in the network and $D$ is the destinations, it is assumed that there is no direct contact between the users and the destinations and communication occurs via $M_{r}$ relays. Each node is equipped with A single antenna. $K_{a}$ is represented by a binomial arbitrary variable and $\mathrm{P}_{\mathrm{k}}\left(K_{a_{0}}=i\right)=\left(\begin{array}{l}L \\ i\end{array}\right) q^{i}(1-q)^{L-i}$ is its probability, $q$ indicates the probability of the active state a user and it should always be $0 \leq q \leq 1$ and $L$ denotes the number of users. For the proposed system model, the ZF technique is used at the relay nodes and assuming that there is a cooperation between the relay nodes, by this we mean that they have the ability of sharing the sent information from the source and transmitted data [12]. The received signal at the relays is as follows

$$
\boldsymbol{y}_{r}=\sqrt{p_{t}} \boldsymbol{G}_{1} \boldsymbol{x}+\boldsymbol{n}_{g},
$$

where $\sqrt{p_{t}}$ is the users transmit power, $\boldsymbol{G}_{1}$ indicates the channel matrix of the first phase of the communication process (i,e users-to-relays links), this can be written as $\boldsymbol{G}_{1}=\boldsymbol{H}_{1} \boldsymbol{D}_{1}^{1 / 2}$, where $\boldsymbol{H}_{1}$ is the small-scale Rayleigh fading parameters matrix, it is $M \times L$ matrix, where $M$ represents the relay receivers antennas and $L$ is the antennas of the user transmitters, and large-scale of the Rayleigh fading is represented by the diagonal matrix $\boldsymbol{D}_{1}$, which is $L \times L$ matrix. The sent symbols are represented by $\boldsymbol{x}=\left[x_{1}, x_{2}, \ldots, x_{L}\right]^{T}$ and $\boldsymbol{n}_{g}$ indicates the noise at the relays, which is AWGN. We assumed that the channels are independently and identically distributed and subjected to complex Gaussian fading with mean equal to zero and variance equal to one $\sim \mathcal{C N}(0,1)$.

At the second stage of the communication, relays forward a transformation of the received data from the users times the transformation matrix $\boldsymbol{V}$. The date at the end user is defined by

$$
\begin{aligned}
y_{D_{i}}=\sqrt{p_{t}} \boldsymbol{g}_{2 i}^{\mathrm{H}} \boldsymbol{V} \boldsymbol{g}_{1 i} x_{i} \\
\quad+\sqrt{p_{t}} \sum_{k=1, k \neq i}^{K} \boldsymbol{g}_{2 i}^{\mathrm{H}} \boldsymbol{V} \boldsymbol{g}_{1 k} x_{k}+\boldsymbol{g}_{2 i}^{\mathrm{H}} \boldsymbol{V} \boldsymbol{n}_{g}+n_{D_{i}},
\end{aligned}
$$

where, $\boldsymbol{g}_{1 i}$ and $\boldsymbol{g}_{1 k}$ represent the $i^{\text {th }}$ and $k^{t h}$ columns of $\boldsymbol{G}_{1}$, respectively, $\boldsymbol{g}_{2 i}$ indicates the $i^{\text {th }}$ column of $\boldsymbol{G}_{2}$, which is the channel matrix of the second phase of the communication. $n_{D_{i}}$ denotes the destination noise $i$, which is AWGN. If there is an available at the relay nodes between source and destination, we can express the relays transformation matrix as $\boldsymbol{V}=\boldsymbol{G}_{2}\left(\boldsymbol{G}_{2}^{\mathrm{H}} \boldsymbol{G}_{2}\right)^{-1}\left(\boldsymbol{G}_{1}^{\mathrm{H}} \boldsymbol{G}_{1}\right)^{-1} \boldsymbol{G}_{1}^{\mathrm{H}}$. In consonance with the $\mathrm{ZF}$ concept that aimed to combat the users interference:

$$
\boldsymbol{g}_{2 i}^{\mathrm{H}} \boldsymbol{V} \boldsymbol{g}_{1 k}=\psi_{k i},
$$

where $\psi_{k i}=1$ when $k=i$, and 0 otherwise [13]. Therefore, we can rewrite (2) as

$$
\boldsymbol{y}_{D_{i}}=\sqrt{p_{t}} x_{i}+\left[\left(\boldsymbol{G}_{1}^{H} \boldsymbol{G}_{1}\right)^{-1} \boldsymbol{G}_{1}^{H}\right]_{i} \boldsymbol{n}_{g}+n_{D_{i}},
$$

To simplify the equation, let $\boldsymbol{G}_{1}^{\dagger}=\left(\boldsymbol{G}_{1}^{\mathrm{H}} \boldsymbol{G}_{1}\right)^{-1} \boldsymbol{G}_{1}^{\mathrm{H}}$ and we obtain

$$
y_{D_{i}}=\sqrt{p_{t}} x_{i}+\left[\boldsymbol{G}_{1}^{\dagger}\right]_{i} \boldsymbol{n}_{g}+n_{D_{i}} .
$$

The $\mathrm{SINR}_{i}$ of the destination is expressed as

$$
\operatorname{SINR}_{i}=\frac{p_{\mathrm{t}}}{\left\|\left[\boldsymbol{G}_{1}^{\dagger}\right]_{i}\right\|^{2} N_{g}+N_{D}},
$$

where $N_{g}$ represents the noise power at the relay nodes and $N_{D}$ the noise power at destination nodes. Hence,

$$
\operatorname{SINR}_{i}=\frac{\left\|\left[\boldsymbol{G}_{1}^{\dagger}\right]_{i}\right\|^{-2}}{\frac{1}{\gamma_{\mathrm{g}}}+\frac{1}{\gamma_{\mathrm{D}}}\left\|\left[\boldsymbol{G}_{1}^{\dagger}\right]_{i}\right\|^{-2}},
$$

where $\left\|\left[\mathbf{G}_{1}^{\dagger}\right]_{i}\right\|^{-2}$ is subjected to Erlang distribution, with $M-L+1$ shape parameter and $\xi_{k}$ scale parameter [14], $\gamma_{\mathrm{g}}$ indicates the SNR at the relay nodes and $\gamma_{D}$ is the SNR at destination nodes. It is assumed that $\gamma_{g}=\gamma_{D}=\gamma$.

\section{SPECTRAL EFFICIENCY ANALYSis}

The achievable SE of the two-way relay system is derived in this section, and it written as

$$
\zeta=\frac{1}{2} \mathrm{E}\left(\sum_{k_{a}=1}^{K_{a}}\left[\log _{2}\left(1+\operatorname{SINR}_{k_{a}}\right)\right]\right),
$$

where $\left[\log _{2}\left(1+\mathrm{SINR}_{k_{a}}\right)\right]$ denotes the instantaneous SE of a user $k_{a}$. The following equation represents the end-to-end SE of the proposed system

$$
\zeta=\frac{1}{2} \mathrm{E}\left\{K_{a}\left[\log _{2}\left(1+\mathrm{SINR}_{1}\right)\right]\right\}
$$

where $\mathrm{SINR}_{1}$ indicates the first user SINR. The assumption $\left\|\left[\mathbf{G}_{1}^{\dagger}\right]_{i}\right\|^{-2}=\boldsymbol{Y}_{k}$, and by using the definition of the moment 
generation function (MGF), the exact analytical expression of the SE can be expressed as

Corollary 1. Let $x_{1}, \ldots \ldots \ldots, x_{N}, y_{1}, \ldots \ldots ., y_{M}$ be arbitrary random variables $>0$. Then [15, eq. (5)]

$$
\begin{aligned}
& \mathrm{E}\left\{\ln \left(1+\frac{\sum_{n_{0}=1}^{N_{0}} x_{n_{0}}}{\sum_{m_{0}=1}^{M_{0}} y_{m_{0}}+1}\right)\right\}= \\
& \quad \int_{0}^{\infty} \frac{1}{f}\left(1-e^{-f \sum_{n_{0}=1}^{N_{0}} x_{n_{0}}}\right) e^{-f\left(\sum_{m_{0}=1}^{M_{0}} y_{m_{0}}+1\right)} d f .
\end{aligned}
$$

Now, we can re-write (9) as

$$
\zeta=\frac{1}{2} \mathrm{E}\left[K_{a}\left(\log _{2} \mathrm{e}\right) \int_{0}^{\infty} \frac{e^{-f / \gamma}}{\mathrm{f}}\left(1-e^{-\mathrm{f} \boldsymbol{Y}_{k}}\right) e^{-f \boldsymbol{Y}_{k} / \gamma} \mathrm{df}\right]
$$

which can be written as

$$
\begin{aligned}
\zeta=\frac{1}{2} \mathrm{E}\left[K_{a}\left(\log _{2} \mathrm{e}\right)\right. \\
\left.\quad \times \int_{0}^{\infty} \frac{e^{-f / \gamma}}{\mathrm{f}}\left(e^{-f Y_{k} / \gamma}-e^{-f \boldsymbol{Y}_{k}\left(1+\frac{1}{\gamma}\right)}\right) \mathrm{df}\right] .
\end{aligned}
$$

Let $u=\frac{\boldsymbol{Y}_{k}}{\gamma}$, and $v=\boldsymbol{Y}_{k}\left(1+\frac{1}{\gamma}\right)$. Thus we obtain $M_{u}(f)=$ $\mathrm{E}\left[e^{-f Y / \gamma}\right]$ and $M_{v}(f)=\mathrm{E}\left[e^{-f Y_{k}\left(1+\frac{1}{\gamma}\right)}\right]$, where $M_{u}(f)=$ $\mathrm{E}\left[e^{-f u}\right]$ and $M_{v}(f)=\mathrm{E}\left[e^{-f v}\right]$ are the MGFs of $u$ and $v$, respectively. Hence,

$M_{u}\left(f \mid K_{a}\right)=\mathbb{E}\left\{e^{-f z \boldsymbol{Y}_{k} / \gamma} \mid K_{a}\right\}=\left\{\frac{1}{1+f / \gamma}\right\}^{M-K_{a}+1}$

and

$$
\begin{aligned}
& M_{v}\left(f \mid K_{a}\right)= \\
& \mathbb{E}\left\{e^{-f Y\left(1+\frac{1}{\gamma}\right)} \mid K_{a}\right\}=\left\{\frac{1}{1+f\left(1+\frac{1}{\gamma}\right)}\right\}^{M-K_{a}+1}
\end{aligned}
$$

Substituting (13) and (14) into (12), the end-to-end SE is expressed by

$$
\begin{gathered}
\zeta=\frac{1}{2} \mathrm{E}\left\{K _ { a } ( \operatorname { l o g } _ { 2 } \mathrm { e } ) \int _ { 0 } ^ { \infty } \frac { e ^ { - f / \gamma } } { \mathrm { f } } \left\{\left\{\frac{1}{1+\mathrm{f} / \gamma}\right\}^{\mathrm{M}-K_{\mathrm{a}}+1}\right.\right. \\
\left.\left.-\left\{\frac{1}{1+\mathrm{f}\left(1+\frac{1}{\gamma}\right)}\right\}^{\mathrm{M}-K_{a}+1}\right\} d f \mid K_{a}\right\}
\end{gathered}
$$

In order to figure-out the expected value of each part, Equation (16) needs to be expanded and simplified as

$$
\begin{aligned}
& \zeta= \frac{1}{2}\left(\log _{2} \mathrm{e}\right) \\
& \times \int_{0}^{\infty} \frac{e^{-f / \gamma}}{\mathrm{f}}\left\{\mathrm{E}\left\{K_{a}(1+\mathrm{f} / \gamma)^{K_{a}}\left\{\frac{1}{1+\mathrm{f} / \gamma}\right\}^{M+1}\right\}\right. \\
&\left.-\mathrm{E}\left\{K_{a}\left(1+f\left(1+\frac{1}{\gamma}\right)\right)^{K_{a}}\left\{\frac{1}{1+f\left(1+\frac{1}{\gamma}\right)}\right\}^{M+1}\right\}\right\} d f .
\end{aligned}
$$

Corollary 2. The expected value of $\mathrm{E}\left[K_{a}(A)^{K_{a}-1}\right]$, where $K_{a}$ is a binomial random variable with probability $P_{r}\left(K_{a}=\right.$ $i)=\left(\begin{array}{l}L \\ i\end{array}\right) q^{i}(1-q)^{L-i}$ can be found as [16, Eq. (28)]

$$
\mathrm{E}\left[K_{a}(A)^{K_{a}-1}\right]=q L(1-q+q A)^{L-1} .
$$

Therefore, the final expression for overall SE can be expressed as

$$
\begin{aligned}
\zeta=\frac{q L}{2}\left(\log _{2} e\right) \int_{0}^{\infty} \frac{e^{-z / \gamma}}{z}\left\{\left\{\frac{(1+\mathrm{qf} / \gamma)^{L-1}}{(1+\mathrm{f} / \gamma)^{M}}\right\}\right. \\
\left.-\left\{\frac{\left(1+\mathrm{qf}\left(1+\frac{1}{\gamma}\right)\right)^{L-1}}{\left(1+f\left(1+\frac{1}{\gamma}\right)\right)^{M}}\right\}\right\} d f .
\end{aligned}
$$

Now, (18) can be expressed in terms of the weights and abscissas of a Laguerre orthogonal polynomial

$$
\begin{aligned}
\zeta=\frac{q L}{2}\left(\log _{2} e\right) & \sum_{n_{0}=1}^{N_{0}} \frac{\alpha_{n_{0}}}{\beta_{n_{0}}}\left\{\left\{\frac{\left(1+\mathrm{q} \mu_{n_{0}} / \gamma\right)^{L-1}}{\left(1+\mu_{n_{0}} / \gamma\right)^{M}}\right\}\right. \\
& \left.-\left\{\frac{\left(1+\mathrm{q} \mu_{n_{0}}\left(1+\frac{1}{\gamma}\right)\right)^{L-1}}{\left(1+\mu_{n_{0}}\left(1+\frac{1}{\gamma}\right)\right)^{M}}\right\}\right\}+R_{N_{0}},
\end{aligned}
$$

where $\mu_{n_{0}}=\beta_{n_{0}} \gamma, \beta_{n_{0}}$ are Laguerre polynomial sample points factor and $\alpha_{n_{0}}$ is the weights factor of it, tabulated in [17, Eq. (25.4.45)], and the remainder $R_{N_{0}}$, is small for $N_{0} \geq 15$. Thus, (19) an effective numerical valuation for the overall SE.

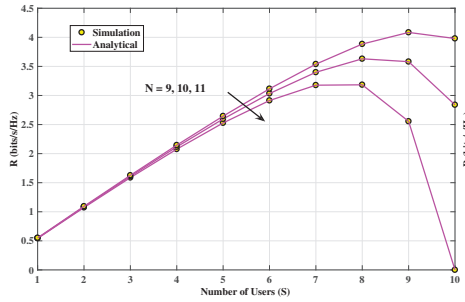

Fig. 2: SE versus different number of users.

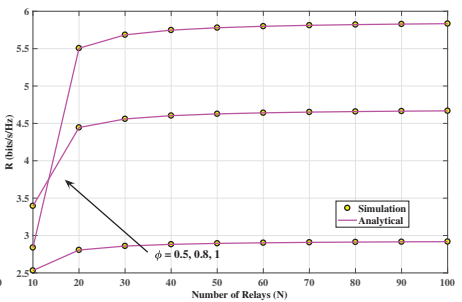

Fig. 3: SE versus different number of relays. 


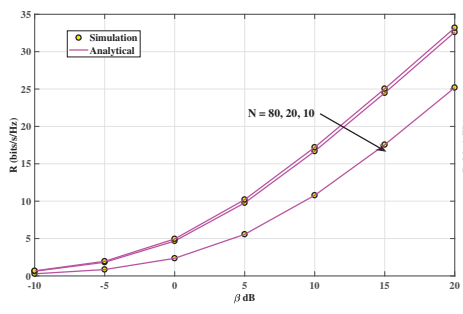

Fig. 4: SE with respect to SNR.

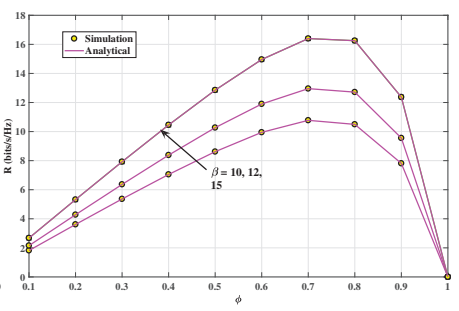

Fig. 5: SE with respect to user activity.

\section{NUMERICAL RESULTS}

In this section, the achievable overall $\mathrm{SE}$ with the use of $\mathrm{ZF}$ technique at the relay nodes is discussed and the numerical results are validated using computer simulations. Fast fading is considered represented by Rayleigh.

In Fig. 2, the overall SE drawn with respect to the users number with different relays number. The analytical results which are obtained by (19) are validated using Monte Carlo simulations. It is clear that the overall SE dramatically increases as the users number increases from 0 to 7 . Nevertheless, for more users, the value of the overall SE relies on the number of relays as seen when $M$ increases from 9 to 11 . When the number of users is higher than the relays number, this will result in a significant degradation in spatial diversity and increasing of interference at relay nodes, which will have an adverse effect on the performance of the system.

Fig. 3, presents the SE as a function of $M$ with different values of $q$ and 10 users. For points on the plot for which $M \geq L$, the SE increases with both the usage and number of users. It can be seen that when $M$ is considerably higher than the users number $(M>L$, that is $M>30)$ all curves have a very slow increasing with further increase in $M$. However, as the user activity becomes higher, the overall SE of the proposed system enhances substantially. This is due to the spatial diversity remains high if $M>30$ regardless of the users activity level, which can be used to determine the maximum number of antennas required for a given application.

Fig. 4 presents the SE as a function in SNR $(\gamma)$ with different relays numbers and 10 users. The results were expected, SE enhances for any increase of $\gamma$. It is noticeable that there is clear enhancement in the SE of the system when $L=10$ users, and $M$ changes from 10 to 20 . However, there is small improvement in SE when $M$ is changing from 20 to 80 with same value of $L$.

Fig. 5 illustrates the effect of the activity on the SE for different values of SNR when $L=11$ and $M=10$. It is clear that the SE initially increases until the user activity reaches about 0.8 for all SNR curves then it starts decreasing until it becomes 0 at $q=1$ where the active users number is more than the relay nodes number, which leads to more interference.

the destination nodes through multi-relays when every node

\section{CONCLusions.}

In this paper, the performance of the wireless system with a random number of active source nodes communicate with is provided with a single antenna. In order to combat the interference, a ZF technique is implemented at the relay nodes. Analytical expressions for the overall SE are derived and we examined the effect of different system parameters on its performance. It was revealed that the analytical results and the computer simulations were in a perfect agreement. The results showed that the SE improves as the users number in the network increases. However, as the users number becomes close to the relays number, the SE starts decreasing. Furthermore, increasing the total relays number in the system can positively affect the system performance. The ZF technique, which is implemented at the relay nodes, can effectively be utilized to combat the users interference.

\section{REFERENCES}

[1] A. Alfitouri and K. A. Hamdi, "Performance analysis of adaptive power technique for cooperative diversity communications," in IEEE Wireless Commun. Netw. Conf. (WCNC), March 2017, pp. 1-6.

[2] J. N. Laneman and G. W. Wornell, "Exploiting distributed spatial diversity in wireless networks," in Proc. Allerton Conf. Commun., Control Com-put., Urbana, IL, Sep. 2000.

[3] W. Gheth, K. M. Rabie, B. Adebisi, M. Ijaz, G. Harris, and A. Alfitouri, "Hybrid power-line/wireless communication systems for indoor applications," in 2018 11th IEEE Int. Symp. Commun. Syst., Netw. and Digit. Signal Process.(CSNDSP), Jul 2018, pp. 1-6.

[4] W. Gheth, K. M. Rabie, B. Adebisi, M. Ijaz, and G. Harris, "Performance analysis of integrated power-line/visible-light communication systems with af relaying," in IEEE Global Commun. Conf. (GLOBECOM), 2018.

[5] A. Wittneben and I. Hammerstrom, "Multiuser zero forcing relaying with noisy channel state information [wireless ad hoc network applications]," in IEEE Wireless Commun. Netw. Conf. (WCNC), vol. 2, 2005, pp. 1018-1023 Vol. 2.

[6] A. Alfitouri and K. A. Hamdi, "Amplify-and-forward gateway with zeroforcing in multiuser environments," IEEE Trans. Veh. Technol., vol. 66 no. 6, pp. 4954-4966, June 2017.

[7] _ - "User selection scheme for amplify-and-forward relaying with zero forcing," in 2016 IEEE Online Conference on Green Communications (OnlineGreenComm), Nov 2016, pp. 33-38.

[8] S. Berger and A. Wittneben, "Cooperative distributed multiuser MMSE relaying in wireless ad-hoc networks," in Signals, Systems and Computers, 2005. Conference Record of the Thirty-Ninth Asilomar Conference on, 2005, pp. 1072-1076.

[9] J. Xue, M. Sellathurai, T. Ratnarajah, and Z. Ding, "Performance analysis for multi-way relaying in rician fading channels," IEEE Trans. Wireless Commun., vol. 63, no. 11, pp. 4050-4062, 2015.

[10] S. Ikki and M. Ahmed, "Performance of cooperative diversity using equal gain combining (EGC) over Nakagami-m fading channels," IEEE Trans. Commun., vol. 8, no. 2, pp. 557-562, Feb. 2009.

[11] Y. Jing and H. Jafarkhani, "Single and multiple relay selection schemes and their achievable diversity orders," IEEE Trans. Commun., vol. 8, no. 3, pp. 1414-1423, Mar. 2009.

[12] M. Torabi and J.-F. Frigon, "Semi-orthogonal relay selection and beamforming for amplify-and-forward MIMO relay channels," in IEEE Wireless Commun. Netw. Conf. (WCNC), Mar. 2008, pp. 48-53.

[13] H. Suraweera, H. Q. Ngo, T. Duong, C. Yuen, and E. Larsson, "Multipair amplify-and-forward relaying with very large antenna arrays," in IEEE Int. Commun. Conf. (ICC), Jun. 2013, pp. 4635-4640.

[14] H. Q. Ngo, M. Matthaiou, T. Duong, and E. Larsson, "Uplink performance analysis of multicell MU-SIMO systems with ZF receivers," IEEE Trans. Veh. Technol., vol. 62, no. 9, pp. 4471-4483, Nov. 2013.

[15] K. Hamdi, "A useful lemma for capacity analysis of fading interference channels," IEEE Trans. Commun., vol. 58, no. 2, pp. 411-416, Feb. 2010 .

[16] A. Alfitouri and K. A. Hamdi, "Multiple-access capabilities of a common gateway," IEEE Trans. Veh. Technol., vol. 66, no. 6, pp. 5148-5159, June 2017.

[17] M. Abramowitz and I. A. Stegun, Handbook of Mathematical Functions. New York, NY, USA: Dover, 1965. 